\title{
Oncogenic CXCL10 signalling drives metastasis development and poor clinical outcome
}

\author{
S C Wightman ${ }^{1,4}$, A Uppal ${ }^{1,4}$, S P Pitroda ${ }^{2,3,4}$, S Ganai ${ }^{1}$, B Burnette ${ }^{2}$, M Stack ${ }^{1}$, G Oshima ${ }^{1}$, S Khan ${ }^{1}$, \\ X Huang ${ }^{2,3}$, M C Posner ${ }^{1,3}$, R R Weichselbaum ${ }^{2,3,5}$ and N N Khodarev ${ }^{\star 2,3,5}$ \\ ${ }^{1}$ Department of Surgery, University of Chicago, Chicago, IL 60637, USA; ${ }^{2}$ Department of Radiation and Cellular Oncology, \\ University of Chicago, Chicago, IL 60637, USA and ${ }^{3}$ Ludwig Center for Metastasis Research, University of Chicago, 5841 South \\ Maryland Avenue, MC 9006, Chicago, IL 60637, USA
}

Background: The CXCL10/CXCR3 signalling mediates paracrine interactions between tumour and stromal cells that govern leukocyte trafficking and angiogenesis. Emerging data implicate noncanonical CXCL10/CXCR3 signalling in tumourigenesis and metastasis. However, little is known regarding the role for autocrine CXCL10/CXCR3 signalling in regulating the metastatic potential of individual tumour clones.

Methods: We performed transcriptomic and cytokine profiling to characterise the functions of CXCL10 and CXCR3 in tumour cells with different metastatic abilities. We modulated the expression of the CXCL10/CXCR3 pathway using shRNA-mediated silencing in both in vitro and in vivo models of B16F1 melanoma. In addition, we examined the expression of CXCL10 and CXCR3 and their associations with clinical outcomes in clinical data sets derived from over 670 patients with melanoma and colon and renal cell carcinomas.

Results: We identified a critical role for autocrine CXCL10/CXCR3 signalling in promoting tumour cell growth, motility and metastasis. Analysis of publicly available clinical data sets demonstrated that coexpression of CXCL10 and CXCR3 predicted an increased metastatic potential and was associated with early metastatic disease progression and poor overall survival.

Conclusion: These findings support the potential for CXCL10/CXCR3 coexpression as a predictor of metastatic recurrence and point towards a role for targeting of this oncogenic axis in the treatment of metastatic disease.

Interferon- $\gamma$-inducible protein CXCL10 (IP-10) is a small $(10 \mathrm{kDa})$ secretable protein in the CXC subfamily of cytokines. Consistent with known functions of CXC cytokines, CXCL10 mediates leukocyte trafficking, adaptive immunity, inflammation, haematopoiesis and angiogenesis (Groom and Luster, 2011; Liu et al, 2011; Zlotnik and Yoshie, 2012). The CXCL10 induces the chemotaxis of various subtypes of leukocytes including $\mathrm{T}$ and B lymphocytes, NK cells, dendritic cells and macrophages (Luster and Ravetch, 1987; Loetscher et al, 1998; Qin et al, 1998; Lo et al, 2010). The CXCL10 signals through the G-protein-coupled seven-transmembrane receptor CXCR3 (Clark-Lewis et al, 2003; Billottet et al, 2013). In human cells, CXCR3 exists in three major isoforms - CXCR3-A, CXCR3-B and CXCR3-alt - that are associated with alternative splicing and potentially different functions (Clark-Lewis et al, 2003; Liu et al, 2011). Signalling occurs through the recruitment of GTP-binding proteins with downstream activation of multiple pathways involved in the regulation of cell survival, proliferation and motility. The CXCR3-A has been associated with pro-survival functions, whereas

\footnotetext{
*Correspondence: Dr NN Khodarev; E-mail: n-khodarev@uchicago.edu

${ }^{4}$ These authors contributed equally to this work.

${ }^{5}$ These authors contributed equally to this work.
}

Received 12 December 2014; revised 30 April 2015; accepted 6 May 2015; published online 4 June 2015 
CXCR3-B is thought to mediate suppressive effects on cell growth and migration; however, these effects are cell and stimuli specific (Liu et al, 2011; Wu et al, 2012; Utsumi et al, 2014).

Recent data implicate CXCL10 in tumourigenesis through different mechanisms. The CXCL10 produced by tumour or host cells can recruit CXCR3 + tumour-infiltrating T cells and NK cells associated with tumour suppression (Luster and Leder, 1993; Wenzel et al, 2005; Fujita et al, 2009; Harlin et al, 2009; Dengel et al, 2010; Antonicelli et al, 2011; Wennerberg et al, 2014). In contrast, CXCR3 + tumour clones, as compared with CXCR3 - clones, have an increased propensity to metastasise potentially because of increased ligand signalling from the premetastatic niche (Kawada et al, 2004; Walser et al, 2006; Kawada et al, 2007; Monteagudo et al, 2007; Zipin-Roitman et al, 2007; Cambien et al, 2009; Ma et al, 2009). Little is known about the metastatic abilities of tumour clones coexpressing CXCL10 and CXCR3 and the effects of constitutively activated autocrine signalling on the development and phenotypic properties of metastatic cells invading the bloodstream.

In the current report, using melanoma tumour cells with different metastatic abilities we demonstrate that the CXCL10/CXCR3 axis expressed by melanoma cells independent of the host microenvironment is sufficient for the regulation of melanoma metastasis to the lung. In addition, analysis of currently available expressional and clinical data sets demonstrates that expression of the CXCL10/CXCR3 axis is associated with increased metastatic potential in melanoma, as well as in colon and renal cell carcinoma patients. These data support the potential for CXCL10/CXCR3 coexpression to be used not only as a predictor for metastatic disease progression, but also as a target for directed therapies in patients with metastatic disease.

\section{MATERIALS AND METHODS}

Cell culture. The generation of $\mathrm{B} 16 \mathrm{~F} 1$ melanoma cell line derivatives with differing metastatic potentials (P2M3C: high potential; P2M5B: low potential) has been previously described (Khodarev et al, 2009). Cells were cultured in RPMI-1640 media (Life Technologies Corporation, Grand Island, NY, USA) supplemented with $10 \%$ fetal bovine serum, $100 \mathrm{U} \mathrm{ml}^{-1}$ penicillin and $100 \mathrm{mg} \mathrm{ml}^{-1}$ streptomycin. All cell lines were maintained in culture with $5 \% \mathrm{CO}_{2}$ at $37^{\circ} \mathrm{C}$.

Animal studies. Tumour cells $\left(10^{5}\right.$ in $100 \mu \mathrm{l}$ PBS $)$ were injected via tail vein into $\mathrm{C} 57 \mathrm{BL} / 6$ or athymic nude mice ( $n=5$ per group) at 5-6 weeks of age (Harlan, Madison, WI, USA). Mice were killed at specified time points and surface lung tumours were manually counted. The care and treatment of experimental animals was in accordance with the institutional guidelines at the University of Chicago.

Proliferation experiments. Primary tumours were established by injection of tumour cells $\left(5 \times 10^{5}\right.$ in $\left.100 \mu \mathrm{l} \mathrm{PBS}\right)$ into the hind limbs of C57BL/6 mice ( $n=8$ per group). Tumour volumes were serially measured and calculated assuming a spherical tumour volume. Cells were also plated $\left(5 \times 10^{4}\right.$ cells per well in 6-well plates or $2 \times 10^{4}$ cells per well in 24 -well plates) in triplicate to assess in vitro growth rate. Cell count was manually determined using a cytometer.

Gene expression analysis. The P2M3C and P2M5B cells grown in culture and as lung metastases were collected in cell lysis buffer and homogenised. RNA was isolated using the RecoverAll Total Nucleic Acid Isolation kit (Ambion, Austin, TX, USA). Three representative in vivo samples for $\mathrm{P} 2 \mathrm{M} 3 \mathrm{C}$ lung metastases were generated using 15 homogenised metastases for each sample obtained from 3 individual mice. Similarly, three in vivo samples for P2M5B cells were generated using 5 homogenised metastases for each sample obtained from 3 to 5 individual mice. The RNA (100 ng) was labelled as per the manufacturer's instructions and profiled with the Illumina Mouse WG6v2 array in triplicate using the iSCAN software (Illumina, Inc., San Diego, CA, USA). Background subtraction, quantile normalisation and log transformation was performed across arrays using the limma Bioconductor package (Smyth, 2005). Differential gene expression between groups was determined using multiple linear regression via the limma package with a Bonferroni post hoc multiplicity correction (corrected $P$-value $\leqslant 0.05$ ) and a fold-change threshold of 1.5. Data were deposited in Gene Expression Omnibus (GEO, accession number pending). Ingenuity Pathway Analysis (IPA, Redwood City, CA, USA) was used for functional gene annotation. Significance was determined using Fisher's exact $P$-value of $\leqslant 0.05$.

Mouse cytokine antibody array. Samples were obtained from $\mathrm{P} 2 \mathrm{M} 3 \mathrm{C}$ and $\mathrm{P} 2 \mathrm{M} 5 \mathrm{~B}$ cell lines grown in vitro and in vivo. Three in vitro samples for each cell line were generated by plating cells $\left(2.5 \times 10^{5}\right)$ in a 6 -well plate in triplicate. At $48 \mathrm{~h}$, culture media were collected $(2 \mathrm{ml})$ and combined for triplicate samples. In vivo samples were generated by collecting five homogenised metastases for each cell line from three individual mice and pooling equal quantities of homogenised material from each mouse. Samples were analysed with the Mouse Cytokine Antibody Array Panel A that measures 40 different cytokines (R\&D Systems, Minneapolis, MN, USA). The density of the arrays was normalised to in assay controls and measured with ImageJ (version 1.46r software; http:// imagej.nih.gov/ij/).

Interferon- $\gamma$ stimulation. Tumour cells were seeded overnight on a 96-well plate $\left(2 \times 10^{4}\right.$ cells per well in RPMI). Recombinant mouse interferon $-\gamma$ (R\&D Systems) was added at either 1000 or 5000 Units $\mathrm{ml}^{-1}$ for 24 or $48 \mathrm{~h}$ and media were collected. Samples were assayed in triplicate using a Quantikine ELISA kit for murine CXCL10 (R\&D Systems).

The shRNA-mediated gene silencing. Stable CXCL10 gene suppression was performed using TRC Lentiviral shRNA clones TRCN0000068210 (sequence: 5'-TAGATTCCGGATTCAGACATC-3'; referred as CXCL10 KD \#1) and TRCN0000068212 (sequence: $5^{\prime}$-TTGATGGTCTTAGATTCCGGA-3'; referred as CXCL10 KD \#2) (Dharmacon, Pittsburgh, PA, USA). Stable CXCR3 gene suppression was achieved using TRC Lentiviral shRNA clones TRCN0000027391 (sequence: 5'-TTCTCTCCGTG AAGATGACGG-3'; referred as CXCR3 KD \#1) and TRCN000002 7383 (sequence: 5'-TTTCTCGACCACAGTTGCGGG-3'; referred as CXCR3 KD \#2). Scrambled shRNAs served as nontargeting controls. Suppression was confirmed using western blot analysis and the Quantikine ELISA kit for murine CXCL10 (R\&D Systems).

Western blot analysis. Total cellular protein was extracted and normalised as previously described (Khodarev et al, 2004). Protein concentrations were adjusted to $1 \mathrm{mg} \mathrm{ml}^{-1}$ and equal amounts of protein were loaded in each well. For total CXCL10, CXCR3 and ERK proteins, $20-25 \mu \mathrm{g}$ of proteins per well were loaded, whereas for phosphorylated proteins (pERK), 30-35 $\mu$ g of proteins per well were loaded. Proteins were separated on $7.5 \%-10 \%$ SDS-PAGE and transferred to polyvinylidene difluoride (PVDF) membranes. For loading control, we used antibodies for $\beta$-actin and $\alpha$-tubulin (sc-47778 and sc-12462-R; Santa Cruz, Santa Cruz, CA, USA). The CXCL10 and CXCR3 antibodies were purchased from R\&D Systems (AF-466-NA and MAB160-100), ERK from Santa Cruz (sc-1647) and pERK from Cell Signaling (Danvers, MA, USA). Images were quantified using ImageJ by integration of pixel values across the area of specific bands.

Migration, invasion and adhesion assays. Costar Transwell Permeable $8.0 \mu \mathrm{m} / 6.5 \mathrm{~mm}$ membranes 3422 (Corning, Corning, 
NY, USA) were coated with either rat tail type I collagen $(200 \mu$ l of $100 \mu \mathrm{g} \mathrm{ml}^{-1}$ mixture in PBS) and 354236 (Corning, Tewksbury, MA, USA) for migration assays or Growth Factor Reduced and Phenol Red-Free Matrigel (200 $\mu$ l of $1: 40$ dilution in PBS) for invasion assays and allowed to harden overnight. Then, PBS was removed from the inserts and replaced with $2 \times 10^{5}$ tumour cells in $200 \mu \mathrm{l}$ RPMI-1640 media without additives. Next, $500 \mu \mathrm{l}$ RPMI-1640 media supplemented with $10 \%$ fetal bovine serum, $100 \mathrm{U} \mathrm{ml}^{-1}$ penicillin and $100 \mathrm{mg} \mathrm{ml}^{-1}$ streptomycin were added into each main well chamber causing a serum gradient. After 4-6 h, the media inside and outside of the insert were carefully removed. The inside of the insert was carefully scraped with a sterile polyester tipped applicator to remove any nonmigrating cells. The insert was placed in $100 \mu \mathrm{l}$ of $4 \%$ paraformaldehyde in PBS and allowed to sit for $15 \mathrm{~min}$. Each insert was then rinsed twice in a 24-well filled with $600 \mu \mathrm{l}$ PBST (500 ml PBS with $250 \mu$ l Tween 20 (Sigma-Aldrich, St Louis, MO, USA)) for $2 \mathrm{~min}$. Each insert was then coated with $100 \mu \mathrm{l}$ of a $1: 10004^{\prime}, 6$-diamidino-2-phenylindole dihydrochloride (DAPI, Life Technologies Corporation) solution in PBS. This was allowed to sit for $20 \mathrm{~min}$ and then each insert was rinsed twice in $600 \mu \mathrm{l}$ PBST for $2 \mathrm{~min}$. Any remaining solution was shaken off and the inserts were imaged at $\times 20$ magnification under UV light and 3 images per insert ( 9 per well) were taken. The number of cells present on each image was counted and averaged for each cell line.

For adhesion assays, Growth Factor Reduced and Phenol RedFree Matrigel 356231 (Corning, Tewksbury, MA, USA) were thawed in an ice bucket for $30 \mathrm{~min}$. Then, $200 \mu \mathrm{l}$ of a $1: 16000$ dilution of Matrigel was placed in each well of a 96-well plate. The plates were incubated overnight to allow for hardening. The following day, the PBS was removed from the 96-well plates, leaving only the Matrigel in each well. Cells $\left(5 \times 10^{4}\right.$ cells per well in PBS) were incubated for $4 \mathrm{~h}$. Each well was washed twice with RPMI-1640 media and then filled with $100 \mu \mathrm{l}$ media and $20 \mu \mathrm{l}$ CellTiter-Blue (Promega, Madison, WI, USA) and allowed to incubate at $37^{\circ} \mathrm{C}$ in $5 \% \mathrm{CO}_{2}$ for $1 \mathrm{~h}$. The relative fluorescence level of each well was then measured and averaged across 10 wells per cell line.

Clinical database analysis. Multiple clinical data sets were examined to assess the relationship between CXCL10 and CXCR3 gene expression, metastatic development and survival in three types of human cancer. The data sets are listed in Supplementary Table S1. Expression was defined as a relative gene expression value greater than the median value across samples. Coexpression denoted expression of both CXCL10 and CXCR3 in a given sample. Rapid metastatic recurrence was defined as a distant metastasis recurrence-free interval less than the median distant metastasis recurrence-free interval across patients. Rapid death was defined as an overall survival less than the median overall survival across patients.

Statistical analysis. Data are presented as mean \pm s.e.m. Twotailed Student's $t$-tests were used to determine differences between means. A $P$-value of $\leqslant 0.05$ determined statistical significance.

\section{RESULTS}

Metastatic ability of tumour cell clones is independent of growth rate. We previously described the derivation of a panel of B16F1 tumour cell lines with different capacities to colonise the lung (Khodarev et al, 2009). In the current study, we utilised two B16F1 derivatives with high and low metastatic potentials, designated $\mathrm{P} 2 \mathrm{M} 3 \mathrm{C}$ and $\mathrm{P} 2 \mathrm{M} 5 \mathrm{~B}$, to investigate the potential molecular mechanisms contributing to the observed metastatic phenotypes. At 4 weeks following tail vein injection, P2M3C cells rapidly colonised the lungs of syngeneic $\mathrm{C} 57 \mathrm{BL} / 6$ mice with a mean \pm s.e.m. number of $127.3 \pm 13.9$ metastases, whereas P2M5B cells formed 45-fold fewer lung metastases with a mean number of $2.8 \pm 2$ metastases $(P=0.011$, Figure $1 \mathrm{~A}$ and $\mathrm{B})$. At 6 weeks following tail vein injection, the mean number of P2M5B lung metastases had not significantly increased when compared with that at 2 or 4 weeks; however, all C57BL/6 mice injected with P2M3C cells had succumbed to death because of metastatic tumour burden (Figure 1B). To assess whether the high metastatic potential of $\mathrm{P} 2 \mathrm{M} 3 \mathrm{C}$ cells was due to an increased growth rate, tumour cells were injected into the hind limbs of C57BL/6 mice (Figure 1C) or grown in tissue culture (Figure 1D). These experiments showed no significant differences in growth kinetics either in vivo or in vitro between $\mathrm{P} 2 \mathrm{M} 3 \mathrm{C}$ and $\mathrm{P} 2 \mathrm{M} 5 \mathrm{~B}$ cells. In addition, we tested the hypothesis that P2M3C cells have increased tumour growth in the lung microenvironment as compared with P2M5B cells. To this end, we compared the sizes of individual lung metastases produced by each cell line, a measure of their tumour growth potential. Consistent with the previous results, this analysis demonstrated no increase in lung colony size in P2M3C cells as compared with P2M5B cells (Supplementary Figure S1). Taken together, these results supported a model in which two tumour cell derivatives exhibited differential abilities to colonise the lung microenvironment that, at least in large part, is independent of growth rate.

High metastatic potential is associated with increased CXCL10 secretion. To assess the molecular differences between tumour cells with high and low metastatic phenotypes, we performed gene expression profiling of $\mathrm{P} 2 \mathrm{M} 3 \mathrm{C}$ and $\mathrm{P} 2 \mathrm{M} 5 \mathrm{~B}$ cells grown in vitro and in vivo from macroscopically resected lung colonies. Using Illumina Mouse WG6v2 gene expression arrays, we identified 153 differentially expressed genes between the two cell lines grown in vitro, of which 117 genes were overexpressed and 36 were suppressed in P2M3C relative to P2M5B. Similarly, we identified 230 differentially expressed genes between in vivo metastatic lesions in P2M3C as compared with P2M5B, of which 167 were overexpressed and 63 were suppressed. Consistent with the observed differences in metastatic phenotypes between P2M3C and P2M5B cells, a large number of pathways involved in 'Cellular Movement' were significantly overexpressed in P2M3C cells and lung metastases as compared with corresponding P2M5B cells (Supplementary Tables S2 and S3). Overall, 30 differentially expressed genes were common to both the in vitro and in vivo conditions and, therefore, considered intrinsic tumour genes. Of these, 25 genes were upregulated and 5 were downregulated in P2M3C relative to P2M5B (Figure 2A and Supplementary Table S4).

Among the differentially expressed genes, CXCL10 was overexpressed by two-fold in P2M3C cells as compared with P2M5B cells $(P=0.0004$, Figure 2B). The CXCL10, also known as interferon- $\gamma$-induced protein 10 , is secreted by several cell types in response to host-derived interferon- $\gamma$ and has known roles in immune modulation and antitumour activity. Given these recognised functions of CXCL10, we explored a potentially unexpected role for CXCL10 in mediating the metastatic phenotype. We measured the secretion of 40 different cytokines by P2M3C and P2M5B cells using the Mouse Cytokine Antibody Array Panel A. These data demonstrated that CXCL10 was the most differentially secreted cytokine by P2M3C cells as compared with P2M5B cells (Figure 2C and D). We found similar results, although to a lesser extent, from P2M3C and P2M5B lung metastases. We also quantified the induction of CXCL10 secretion as a function of exogenous interferon- $\gamma$ stimulation in P2M3C and P2M5B cells. At 24 and $48 \mathrm{~h}$ after stimulation with varying doses of interferon- $\gamma, \mathrm{P} 2 \mathrm{M} 3 \mathrm{C}$ cells consistently secreted significantly greater levels of CXCL10 as compared with P2M5B cells (Supplementary Figure S2). These results demonstrated that the increased production of tumour cell-derived CXCL10 is associated with a 
A

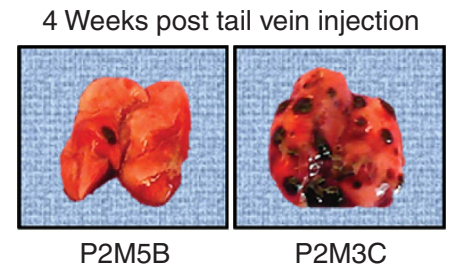

C

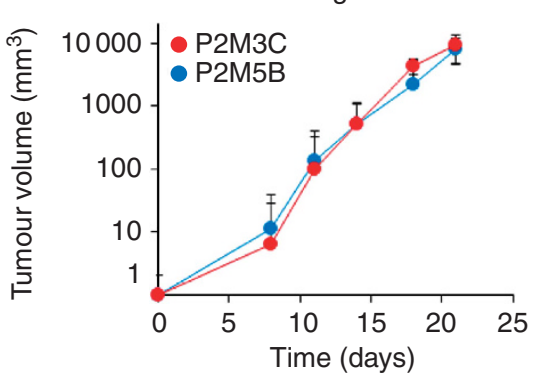

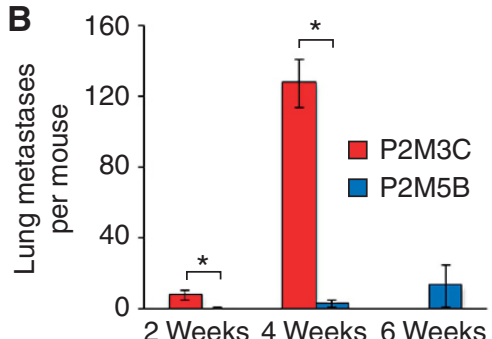

D

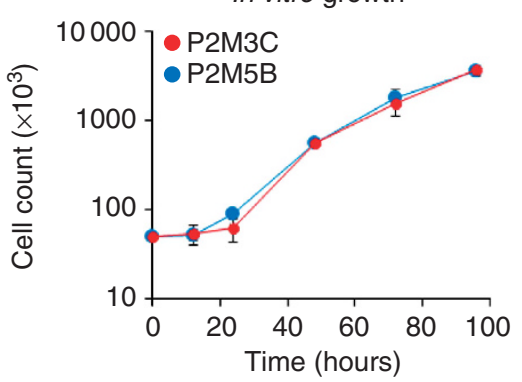

Figure 1. Metastatic melanoma derivatives differ in lung colonisation ability independent of growth rate. (A) Representative images of lung colonisation at 4 weeks following tail vein injection of P2M5B (left) and P2M3C (right) tumour cells into C57BL/6 mice. (B) Quantification of surface lung colonies at 2, 4 and 6 weeks after tail vein injection of P2M3C and P2M5B cells. All C57BL/6 mice injected with P2M3C cells had died by 6 weeks because of tumour burden. (C) Tumour volume of P2M3C and P2M5B cells established as hind limb tumours in C57BL/6 mice. (D) Cell growth of $\mathrm{P} 2 \mathrm{M} 3 \mathrm{C}$ and $\mathrm{P} 2 \mathrm{M} 5 \mathrm{~B}$ in tissue culture. Data represent mean \pm s.e.m. $(n=3)$. ${ }^{\star} P \leqslant 0.05$.

high metastatic potential and suggest a role for host-derived interferon $-\gamma$ in the induction of CXCL10 expression in metastatic tumour cells.

CXCL10/CXCR3 axis promotes tumour cell metastasis. The predominant actions of CXCL10 on target cells occur through the binding of its cell surface cognate receptor CXCR3. The CXCR3 also binds other CXC cytokines, including CXCL9, CXCL11 and CXCL4; however, none of these CXC cytokines were differentially expressed in P2M3C cells as compared with P2M5B cells. In this context, we examined whether the CXCL10/CXCR3 axis directly mediates the metastatic phenotype of tumour cells. Using lentiviral-based shRNA constructs we stably suppressed CXCL10 or CXCR3 in P2M3C and P2M5B cells. As determined by western blot analysis, shRNA-mediated silencing led to significant suppression of CXCL10 and CXCR3 protein levels (Figure 3A). Suppression of CXCL10 also led to a significant reduction in CXCL10 secretion in both P2M3C and P2M5B cells $(P<0.001$, Figure 3B).

Suppression of CXCL10 in P2M3C cells significantly decreased lung colonisation after tail vein injection into $\mathrm{C} 57 \mathrm{BL} / 6$ mice by approximately three-fold (KD \#1: $27.8 \pm 7.9$; $\mathrm{KD} \# 2$ : $26.7 \pm 6.4$ ) as compared with nontargeting control cells $(89.7 \pm 5.4, P<0.001$, Figure $3 \mathrm{C}$ ). Interestingly, the number of lung colonies in $\mathrm{P} 2 \mathrm{M} 3 \mathrm{C}$ cells after suppression of CXCL10 was similar to that of P2M5B control cells. Knockdown of CXCL10 in P2M5B cells also led to a significant reduction in lung colonisation ( $\mathrm{KD} \# 1$ : $9.4 \pm 2.3$; $\mathrm{KD} \# 2$ : $2.8 \pm 0.06)$ as compared with nontargeting control cells (25.4 \pm 4.1 , $P<0.01$, Figure 3C). We obtained similar results when shCXCL10 $\mathrm{P} 2 \mathrm{M} 3 \mathrm{C}$ and P2M5B cells were injected into the tail veins of athymic nude mice, suggesting that the effect of CXCL10 on metastatic potential in both $\mathrm{P} 2 \mathrm{M} 3 \mathrm{C}$ and $\mathrm{P} 2 \mathrm{M} 5 \mathrm{~B}$ cells was largely independent of an intact host immune response on metastatic tumour cells (Supplementary Figure S3A and B). Suppression of CXCR3 led to a similar phenotypic reduction in lung colonisation in both $\mathrm{P} 2 \mathrm{M} 3 \mathrm{C}$ and $\mathrm{P} 2 \mathrm{M} 5 \mathrm{~B}$ cells (Figure 3D). As compared with a nontargeting control (134.4 \pm 14.7$)$, CXCR3 knockdown significantly reduced P2M3C lung metastases (KD \#1: $86.1 \pm 12.2$,
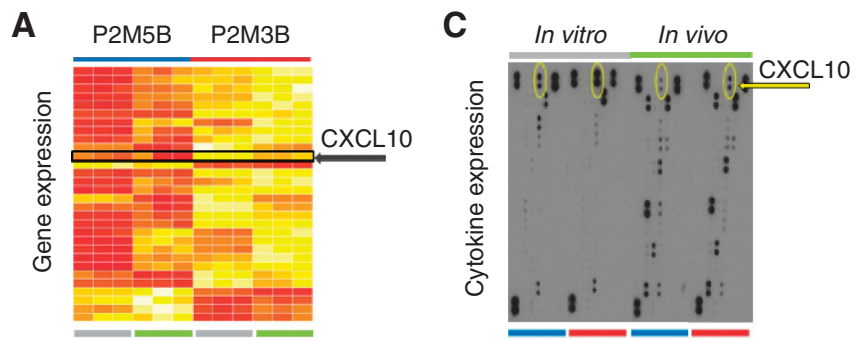

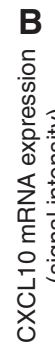
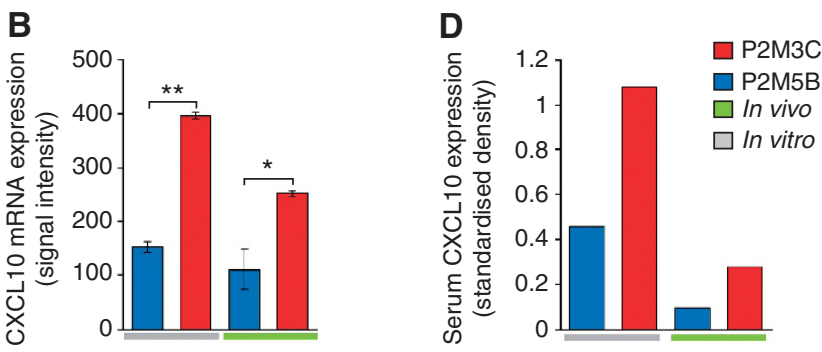

Figure 2. The $\mathrm{P} 2 \mathrm{M} 3 \mathrm{C}$ cells exhibit increased expression and secretion of CXCL10 as compared with P2M5B cells. (A) Heat map of 30 genes differentially expressed between P2M5B and P2M3C cells grown in tissue culture (in vitro: grey line) and as lung metastases (in vivo: green line). Yellow indicates high expression, whereas red denotes low expression. The CXCL10 is indicated by a black arrow. (B) The mRNA expression of CXCL10 as determined by Illumina Mouse WG6v2 gene expression signal intensity values. (C) Densitometry map of secreted murine cytokines produced by pooled samples of P2M5B (blue bar) and P2M3C (red bar) cells in vitro and in vivo as measured using Mouse Cytokine Antibody Array Panel A. The size and density of each dot correlates with the amount of secreted cytokine. The CXCL10 is indicated by a yellow arrow. (D) Quantification of serum CXCL10 protein production relative to in assay controls (standardised density) for P2M5B (blue bar) and P2M3C (red bar) cells grown in vitro (grey line) and in vivo (green line). Data represent mean \pm s.e.m. $(n=3) .{ }^{\star} P \leqslant 0.05$. $\star * P \leqslant 0.001$. 
A

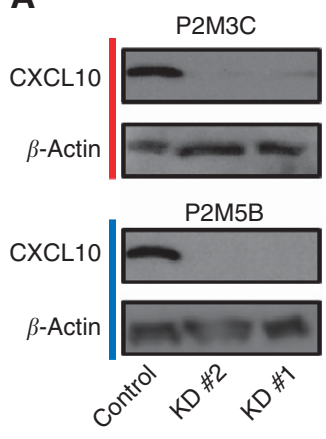

$\mathrm{P} 2 \mathrm{M} 3 \mathrm{C}$

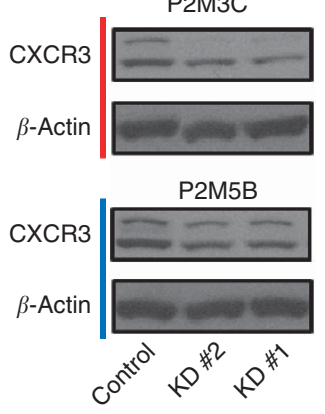

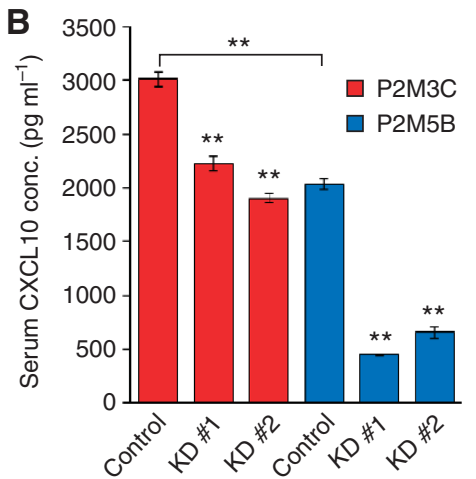

D

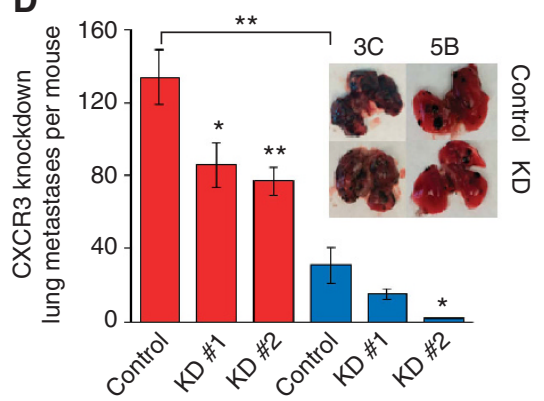

Figure 3. Suppression of the CXCL10/CXCR3 axis reduces lung metastatic colonisation. (A) Stable shRNA-mediated suppression of CXCL10 and CXCR3 as confirmed by western blot analysis. Two shRNA constructs (KD \#1 and KD \#2) were tested for each gene of interest. Nontargeting shRNAs served as controls. $\beta$-Actin served as a protein control. (B) Reduction in serum CXCL10 concentration following shRNA-mediated suppression of CXCL10 in P2M3C and P2M5B cells as measured by ELISA. Quantification of surface lung colonies 3 weeks after tail vein injection in C57BL/6 mice of P2M3C and P2M5B cells transfected with shRNA targeting CXCL10 (C) or CXCR3 (D). Insets display representative images of lung colonisation. ${ }^{*} P \leqslant 0.05 .{ }^{*} P \leqslant 0.01$.

$P=0.03 ; \mathrm{KD} \# 2: 77.3 \pm 7.5, P=0.005)$. In P2M5B cells, suppression of CXCR3 also reduced lung colonisation (KD \#1: $14.7 \pm 2.8$, $P=0.14 ; \mathrm{KD} \# 2: 1.7 \pm 0.3)$ as compared with control cells $(31.3 \pm 9.6, P=0.02)$. To test the functional activity of the CXCL10/CXCR3 axis, we examined ERK1/2 phosphorylation in response to CXCL10 stimulation of P2M3C and P2M5B cell lines (Bonacchi et al, 2001). These data demonstrated increased phosphorylation of ERK1/2 in both cell lines at $4 \mathrm{~h}$ following CXCL10 treatment (Supplementary Figure S4). This result supports active CXCR3-mediated signalling in P2M3C and P2M5B cell lines. Taken together, these data demonstrated that the CXCL10/CXCR3 axis enhances the metastatic phenotype of tumour cells.

CXCL10 regulates cell-autonomous properties of metastatic tumour cells. The CXCL10 has been implicated in several cellular functions including the regulation of cell growth and motility. We examined the effects of CXCL10 in mediating such functions in metastatic tumour cells. Cells were grown either in tissue culture (Figure 4A) or injected into the hind limbs of C57BL/6 mice (Figure 4B). The data showed that suppression of CXCL10 resulted in significant decreases in cell and tumour growth of both P2M3C and P2M5B cell lines, although the effect of CXCL10 suppression was more pronounced in P2M3C as compared with $\mathrm{P} 2 \mathrm{M} 5 \mathrm{~B}$ cells (Figure 4A and B). We also examined the effect of CXCL10 on cellular migration, invasion and adhesion. Control P2M3C cells exhibited increased cell migration (Figure 4C) and adhesion (Figure 4D), but not invasion (Figure 4C), as compared with control P2M5B cells. Suppression of CXCL10 resulted in significant decreases in cell migration, invasion and adhesion properties of $\mathrm{P} 2 \mathrm{M} 3 \mathrm{C}$ cells but only a reduction in cell adhesion of P2M5B cells. Consistent with these results, suppression of CXCR3 resulted in significant decreases in adhesion properties of both cell lines (Supplementary Figure S5). Taken together, these data demonstrated that the CXCL10/CXCR3 axis mediates properties of metastatic tumour cells through regulation of adhesion, invasion and migration phenotypes.

Coexpression of CXCL10/CXCR3 in clinical tumours is associated with metastatic disease. In the context of the above findings, we hypothesised that CXCL10/CXCR3 expression is associated with metastasis in clinical cancer samples. We explored multiple human cancer types for which CXCL10 has been implicated in mediating metastasis, including melanoma, renal cell carcinoma and colon carcinoma. Utilising The Cancer Genome Atlas (CGA) data set consisting of 328 primary and metastatic melanoma samples, we found that CXCL10 and CXCR3 gene expression values were significantly correlated (Pearson's correlation coefficient $r=0.47, P<0.0001$, Figure 5A). We examined primary melanomas and melanoma metastases and found that CXCL10/CXCR3 coexpression occurred more than twice as frequently in melanoma metastases (43\%) as compared with primary melanoma tumours $\left(20 \%, P=0.0016, \chi^{2}\right.$ test, Figure $\left.5 B\right)$. In the CGA data set, information regarding disease recurrence was available for 172 patients, and of these, distant metastasis accounted for $48 \%$ of subsequent disease recurrences during the follow-up period. The incidence of CXCL10/CXCR3 coexpression was $50 \%$ greater in samples with distant metastases at the time of recurrence (left panel, Figure 5C, 60\% vs 40\%, $P=0.050, \chi^{2}$ test). In a subset analysis of patients with known metastatic disease, CXCL10/CXCR3 coexpression rates were 13-fold less in patients who did not develop distant metastasis at the time of recurrence (right panel, Figures $5 \mathrm{C}, P=0.018, \chi^{2}$ test). In a logistic regression analysis of distant metastatic disease recurrence, only CXCL10/ CXCR3 coexpression and nodal involvement were significant covariates on univariate analysis when compared with other traditional clinical variables, including tumour stage, ulceration, mitotic rate and age. On multivariate analysis, coexpression of 

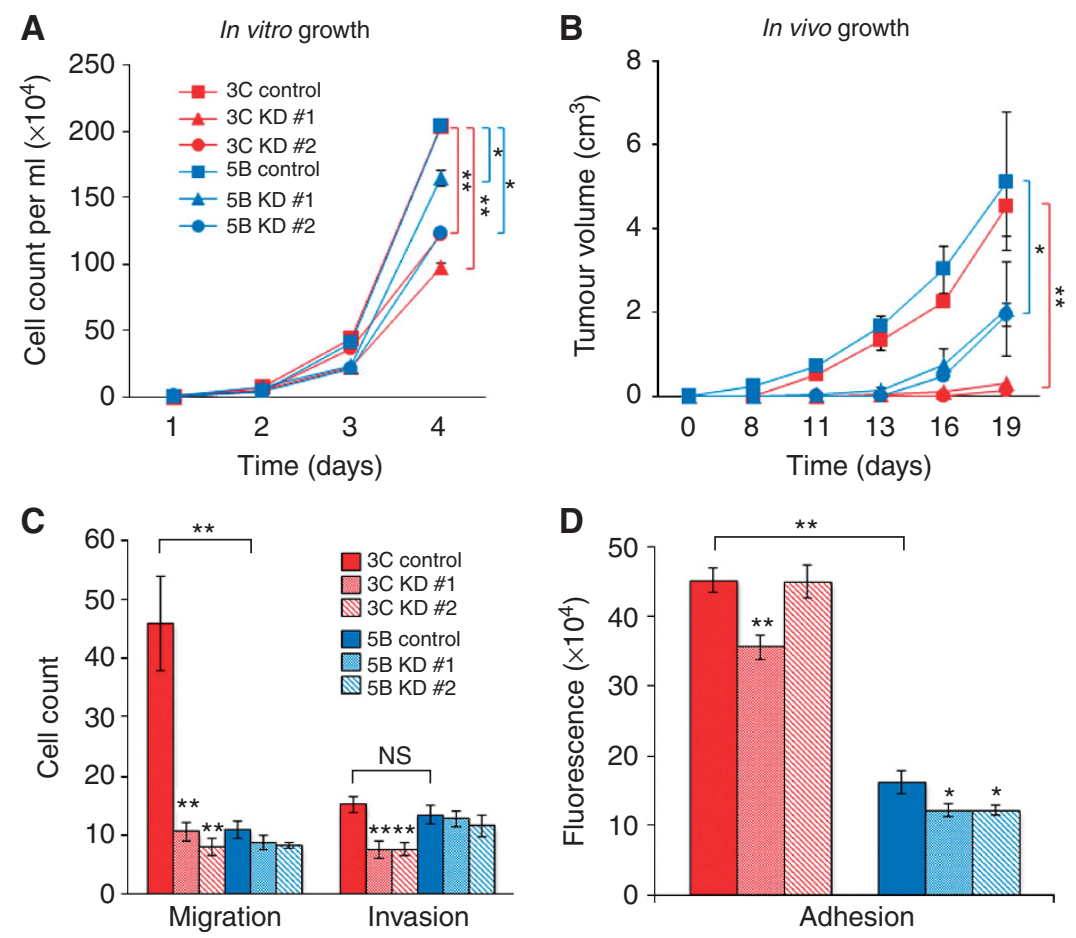

Figure 4. The CXCL10 mediates cell growth and motility. The P2M3C and P2M5B cell growth in tissue culture (A) and as hind limb tumours in C57BL/6 mice (B) after transfection with shRNA targeting CXCL10 (KD \#1 and KD \#2) or a nontargeting control. The P2M3C and P2M5B cell migration (C), invasion $(\mathbf{C})$ and adhesion $(\mathbf{D})$ after shRNA-mediated suppression of CXCL10 as compared with a nontargeting control. Data represent mean \pm s.e.m. $(n=3)$. ${ }^{\star} P \leqslant 0.05$. ${ }^{\star \star} P \leqslant 0.001$. NS indicates no significant difference.
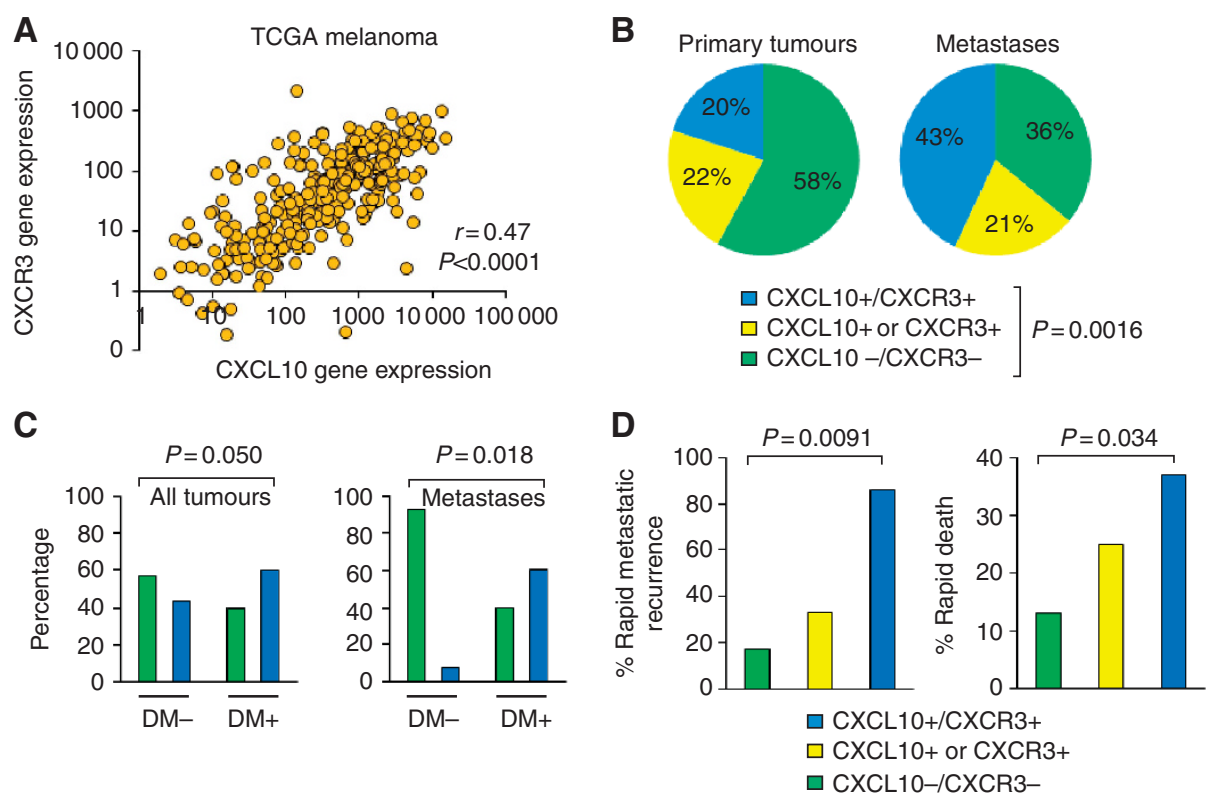

Figure 5. Coexpression of CXCL10/CXCR3 in clinical tumours predicts early metastatic recurrence and reduced survival. (A) Pearson's correlation analysis of CXCL10 and CXCR3 gene expression values derived from The Cancer Genome Atlas (TCGA) data set. Data are plotted on a log-log scale. Each point corresponds to an individual tumour sample $(n=328)$. (B) Frequencies in expression of CXCL10 and/or CXCR3 in primary melanoma tumours $(n=57)$ and melanoma metastases $(n=271)$ in TCGA. Positive gene expression was defined as an expression value above the median value across all samples. (C) Frequencies of CXCL10/CXCR3 coexpression (blue bar) as compared with CXCL10/CXCR3 nonexpressors (green bar) in primary tumours and metastases (left panel) or specifically in metastases (limited to lymph node, in-transit and distant metastases) (right panel) as a function of distant metastatic recurrence (DM). (D) Rapid distant metastatic recurrence (time to distant metastasis recurrence less than group median; $n=19$; left panel) and rapid death (time to death less than group median; $n=76$; right panel) as a function of CXCL10/CXCR3 coexpression. Differences in frequencies were determined using $\chi^{2}$ tests.

CXCL10/CXCR3 remained an independent predictive factor of distant metastatic disease recurrence when lymph node involvement was considered $(P=0.027$, Likelihood Ratio Test,
Supplementary Table S5). Based on these findings, we hypothesised that CXCL10/CXCR3 coexpression in melanoma metastases would be associated with an increased potential for subsequent distant 
metastatic recurrence. We compared CXCL10/CXCR3 coexpression in consecutive recurrent metastases derived from two independent clinical data sets. The results demonstrated that coexpression of CXCL10/CXCR3 was associated with a 2.6- to 5.1fold greater incidence of rapid metastatic recurrence as compared with samples lacking expression of CXCL10 and/or CXCR3 (Figure 5D left panel, $P=0.0091, \chi^{2}$ test). In concert with these findings, CXCL10/CXCR3 coexpression in melanoma metastases was associated with significantly inferior overall survival times for patients with metastatic disease (Figure 5D, right panel, $P=0.034$, $\chi^{2}$ test). Kaplan-Meier survival analysis of distant metastasis-free survival (DMFS) demonstrated significant differences (log-rank $P=0.0050)$ between patient groups based on coexpression of CXCL10 and CXCR3 (Supplementary Figure S6). Taken together, these results demonstrated that co-expression of CXCL10/CXCR3 is independently associated with distant metastatic disease recurrence and inferior patient outcomes.

We extended these findings in clinical data sets of renal cell and colon carcinoma. In primary colon cancers $(n=186)$ and corresponding lung metastases $(n=20)$, CXCL10 and CXCR3 gene expression values were also significantly correlated $(r=0.44$, $P<0.0001$, Supplementary Figure S7A). In addition, CXCL10/ CXCR3 coexpression occurred $30 \%$ more frequently in lung metastases $(70 \%)$ as compared with primary colon cancers $(40 \%$, Supplementary Figure $S 7 B, P=0.010, \chi^{2}$ test). In primary renal cell carcinomas $(n=24)$ and matched lung metastases $(n=24)$, we again found a significant correlation between CXCL10 and CXCR3 gene expression values $(r=0.61, P<0.0001$, Supplementary Figure S8A). Notably, lung metastases exhibiting overexpression of CXCL10 had 1.6-fold higher expression of CXCR3 (Student's $t$-test $P=0.045$ ) and were 2.2 -fold more likely to develop rapid metastatic recurrence as compared with low CXCL10 expressors (78\% vs 36\%, $P=0.036, \chi^{2}$ test, Supplementary Figure S8B).

Taken together, these results demonstrated that CXCL10 and CXCR3 are coexpressed in clinical samples of melanoma as well as renal cell and colon cancers. Both CXCL10 and CXCR3 are coexpressed more frequently in metastases than in primary tumours. Importantly, the coexpression of CXCL10/CXCR3 predicted early metastatic disease recurrence and poor overall survival.

\section{DISCUSSION}

In the current report, we demonstrated that autocrine CXCL10/ CXCR3 signalling in tumour cells with equal growth potential contributes to metastasis primarily through regulation of cellular adhesion, invasion and migration (AIM) properties. The AIM properties of tumour cells are key determinants in the final stages of metastatic colonisation of distant organ sites (Uppal et al, 2015). In multiple clinical data sets derived from patients with melanoma, colon carcinoma or renal cell carcinoma, CXCL10 and CXCR3 were frequently coexpressed in metastases as compared with primary tumours. In addition, coexpression of CXCL10/CXCR3 was associated with rapid metastatic disease progression and poor overall survival.

Traditionally, CXCL10/CXCR3 signalling has been associated with paracrine interactions regulating leukocyte trafficking and angiogenesis (Luster and Ravetch, 1987; Loetscher et al, 1998; Qin et al, 1998; Groom and Luster, 2011; Liu et al, 2011; Zlotnik and Yoshie, 2012). However, recent data have begun to elucidate noncanonical functions of CXCL10 and CXCR3 in the contexts of tumourigenesis and metastasis (Luster and Leder, 1993; Kawada et al, 2004, 2007; Jöhrer et al, 2005; Walser et al, 2006, 2007; Monteagudo et al, 2007; Zipin-Roitman et al, 2007; Cambien et al, 2009; Fujita et al, 2009; Ma et al, 2009; Dengel et al, 2010;
Lo et al, 2010; Antonicelli et al, 2011; Liu et al, 2011; Lee et al, 2012; Wu et al, 2012; Billottet et al, 2013; Murakami et al, 2013; Utsumi et al, 2014; Wennerberg et al, 2014). The CXCL10 has been shown to induce tumour cell migration, invasion and survival in various human cancer cell lines (Clark-Lewis et al, 2003; Kawada et al, 2004; Zipin-Roitman et al, 2007; Lee et al, 2012). Importantly, some of these publications relied on the effects of exogenous CXCL10 rather than intrinsic production of CXCL10 by cancer cells. It was also demonstrated that tumour clones expressing CXCL10, but not CXCL9, grew rapidly as cutaneous tumours because of the lack of a $\mathrm{T}$ cell-mediated antitumour response (Gorbachev et al, 2007). However, the autocrine effects of CXCL10 were not characterised. In concert with these findings, previous reports have demonstrated that CXCR3 is often overexpressed in some tumours as compared with normal tissue. Whether this receptor is regulated in a cell-autonomous tumour-dependent context or is activated by host-derived ligands remains largely unknown. Our data indicate that modulation of the CXCL10/ CXCR3 axis in tumour cells disseminating through the bloodstream leads to consistent effects on metastasis development in both immunocompetent and immunocompromised hosts.

Our experimental data are also consistent with analysis of available clinical data sets and revealed three important features. First, the coexpression of CXCL10/CXCR3 was more likely to be associated with poor clinical outcome as compared with expression of either CXCL10 or CXCR3. In addition, CXCL10 and CXCR3 were significantly correlated in multiple human cancer types. Second, in different data sets derived from various cancer types encompassing more than 670 specimens, there was an enrichment of CXCL10/CXCR3 coexpressing cells in metastatic samples as compared with primary tumours. These data support a critical role for autocrine CXCL10/CXCR3 signalling in the development of metastases. Third, our analysis demonstrated that in distant metastases, CXCL10/CXCR3 coexpression is associated with an increased risk of metastatic disease recurrence. Taken together, these data converge on the direct effects of CXCL10-CXCR3 signalling on the metastatic ability of cancer cells.

Emerging data have also revealed that in certain cellular contexts interferon-stimulated genes (ISGs) may paradoxically mediate oncogenic properties (Weichselbaum et al, 2008; Khodarev et al, 2010; Cheon et al, 2011; Khodarev et al, 2012; Cheon et al, 2013; Kufe, 2013; Boelens et al, 2014). The CXCL10 is a prototypical ISG and a member of the Interferon-Related DNA Damage Signature (IRDS). Genes in this signature are constitutively expressed in different types of cancers and are often associated with metastatic dissemination, poor prognosis and/or resistance to anticancer therapies (Khodarev et al, 2004, 2009, 2012; Weichselbaum et al, 2008; Schultz et al, 2010; Duarte et al, 2012). Specific oncogenic functions of IRDS genes are not well understood; however, many of these genes are well characterised in the context of antiviral responses (Khodarev et al, 2012; Cheon et al, 2013; Sistigu et al, 2014). The data presented in the current report support a role for constitutively expressed CXCL10 as conferring pro-metastatic functions in tumour cells that may, at least in part, explain the association between IRDS and poor prognosis. Taken together, these findings strongly support a strategy to target the CXCL10/CXCR3 axis in the treatment of metastatic disease.

\section{ACKNOWLEDGEMENTS}

We thank Dr Samuel Hellman for his helpful discussions regarding this manuscript. This work was supported in part by The Virginia and DK Ludwig Fund for Cancer Research, Lung Cancer Research Foundation (LCRF), Prostate Cancer Foundation (PCF) and a 
generous gift from the Foglia family. The funders had no role in study design, data collection and analysis, decision to publish or preparation of the manuscript.

\section{CONFLICT OF INTEREST}

The authors declare no conflict of interest.

\section{REFERENCES}

Antonicelli F, Lorin J, Kurdykowski S, Gangloff SC, Le Naour R, Sallenave JM, Hornebeck W, Grange F, Bernard P (2011) CXCL10 reduces melanoma proliferation and invasiveness in vitro and in vivo. $\mathrm{Br} J$ Dermatol 164: $720-728$.

Billottet C, Quemener C, Bikfalvi A (2013) CXCR3, a double-edged sword in tumor progression and angiogenesis. Biochim Biophys Acta 1836: 287-295.

Boelens MC, Wu TJ, Nabet BY, Xu B, Qiu Y, Yoon T, Azzam DJ, TwymanSaint Victor C, Wiemann BZ, Ishwaran H, Ter Brugge PJ, Jonkers J, Slingerland J, Minn AJ (2014) Exosome transfer from stromal to breast cancer cells regulates therapy resistance pathways. Cell 159: 499-513.

Bonacchi A, Romagnani P, Romanelli RG, Efsen E, Annunziato F, Lasagni L, Francalanci M, Serio M, Laffi G, Pinzani M, Gentilini P, Marra F (2001) Signal transduction by the chemokine receptor CXCR3: activation of Ras/ERK, Src, and phosphatidylinositol 3-kinase/Akt controls cell migration and proliferation in human vascular pericytes. J Biol Chem 276: 9945-9954.

Cambien B, Karimdjee BF, Richard-Fiardo P, Bziouech H, Barthel R, Millet MA, Martini V, Birnbaum D, Scoazec JY, Abello J, Al Saati T, Johnson MG, Sullivan TJ, Medina JC, Collins TL, Schmid-Alliana A, SchmidAntomarchi H (2009) Organ-specific inhibition of metastatic colon carcinoma by CXCR3 antagonism. Br J Cancer 100: 1755-1764.

Cheon H, Holvey-Bates EG, Schoggins JW, Forster S, Hertzog P, Imanaka N, Rice CM, Jackson MW, Junk DJ, Stark GR (2013) IFN $\beta$-dependent increases in STAT1, STAT2, and IRF9 mediate resistance to viruses and DNA damage. EMBO J 32(2751): 63.

Cheon H, Yang J, Stark GR (2011) The functions of signal transducers and activators of transcriptions 1 and 3 as cytokine-inducible proteins J Interferon Cytokine Res 31: 33-40.

Clark-Lewis I, Mattioli I, Gong JH, Loetscher P (2003) Structure-function relationship between the human chemokine receptor CXCR3 and its ligands. J Biol Chem 278: 289-295.

Dengel LT, Norrod AG, Gregory BL, Clancy-Thompson E, Burdick MD, Strieter RM, Slingluff Jr CL, Mullins DW (2010) Interferons induce CXCR3-cognate chemokine production by human metastatic melanoma J Immunother 33: 965-974.

Duarte CW, Willey CD, Zhi D, Cui X, Harris JJ, Vaughan LK, Mehta T, McCubrey RO, Khodarev NN, Weichselbaum RR, Gillespie GY (2012) Expression signature of IFN/STAT1 signaling genes predicts poor survival outcome in glioblastoma multiforme in a subtype-specific manner. PLoS One 7: e29653.

Fujita M, Zhu X, Ueda R, Sasaki K, Kohanbash G, Kastenhuber ER, McDonald HA, Gibson GA, Watkins SC, Muthuswamy R, Kalinski P, Okada H (2009) Effective immunotherapy against murine gliomas using type 1 polarizing dendritic cells- significant roles of CXCL10. Cancer Res 69: 1587-1595.

Gorbachev AV, Kobayashi H, Kudo D, Tannenbaum CS, Finke JH, Shu S, Farber JM, Fairchild RL (2007) CXC chemokine ligand 9/monokine induced by IFN-gamma production by tumor cells is critical for T cellmediated suppression of cutaneous tumors. J Immunol 178: 2278-2286.

Groom JR, Luster AD (2011) CXCR3 ligands: redundant, collaborative and antagonistic functions. Immunol Cell Biol 89: 207-215.

Harlin H, Meng Y, Peterson AC, Zha Y, Tretiakova M, Slingluff C, McKee M, Gajewski TF (2009) Chemokine expression in melanoma metastases associated with CD8 + T-cell recruitment. Cancer Res 69: 3077-3085.

Jöhrer K, Zelle-Rieser C, Perathoner A, Moser P, Hager M, Ramoner R, Gander H, Höltl L, Bartsch G, Greil R, Thurnher M (2005) Up-regulation of functional chemokine receptor CCR3 in human renal cell carcinoma. Clin Cancer Res 11: 2459-2465.

Kawada K, Hosogi H, Sonoshita M, Sakashita H, Manabe T, Shimahara Y, Sakai Y, Takabayashi A, Oshima M, Taketo MM (2007) Chemokine receptor CXCR3 promotes colon cancer metastasis to lymph nodes. Oncogene 26: 4679-4688.
Kawada K, Sonoshita M, Sakashita H, Takabayashi A, Yamaoka Y, Manabe T, Inaba K, Minato N, Oshima M, Taketo MM (2004) Pivotal role of CXCR3 in melanoma cell metastasis to lymph nodes. Cancer Res 64: 4010-4017.

Khodarev N, Ahmad R, Rajabi H, Pitroda S, Kufe T, McClary C, Joshi MD, MacDermed D, Weichselbaum R, Kufe D (2010) Cooperativity of the MUC1 oncoprotein and STAT1 pathway in poor prognosis human breast cancer. Oncogene 29: 920-929.

Khodarev NN, Beckett M, Labay E, Darga T, Roizman B, Weichselbaum RR (2004) STAT1 is overexpressed in tumors selected for radioresistance and confers protection from radiation in transduced sensitive cells. Proc Natl Acad Sci USA 101: 1714-1719.

Khodarev NN, Pitroda SP, Beckett MA, MacDermed DM, Huang L, Kufe DW, Weichselbaum RR (2009) MUC1-induced transcriptional programs associated with tumorigenesis predict outcome in breast and lung cancer. Cancer Res 69: 2833-2837.

Khodarev NN, Roach P, Pitroda SP, Golden DW, Bhayani M, Shao MY, Darga TE, Beveridge MG, Sood RF, Sutton HG, Beckett MA, Mauceri HJ, Posner MC, Weichselbaum RR (2009) STAT1 pathway mediates amplification of metastatic potential and resistance to therapy. PLoS One 4: e5821.

Khodarev NN, Roizman B, Weichselbaum RR (2012) Molecular pathways: interferon/stat1 pathway: role in the tumor resistance to genotoxic stress and aggressive growth. Clin Cancer Res 18: 3015-3021.

Kufe DW (2013) MUC1-C oncoprotein as a target in breast cancer: activation of signaling pathways and therapeutic approaches. Oncogene 32: 1073-1081.

Lee JH, Kim HN, Kim KO, Jin WJ, Lee S, Kim HH, Ha H, Lee ZH (2012) CXCL10 promotes osteolytic bone metastasis by enhancing cancer outgrowth and osteoclastogenesis. Cancer Res 72: 3175-3186.

Liu M, Guo S, Stiles JK (2011) The emerging role of CXCL10 in cancer (Review). Oncol Lett 2: 583-589.

Lo BK, Yu M, Zloty D, Cowan B, Shapiro J, McElwee KJ (2010) CXCR3/ ligands are significantly involved in the tumorigenesis of basal cell carcinomas. Am J Pathol 176: 2435-2446.

Loetscher M, Loetscher P, Brass N, Meese E, Moser B (1998) Lymphocytespecific chemokine receptor CXCR3: regulation, chemokine binding and gene localization. Eur J Immunol 28: 3696-3705.

Luster AD, Leder P (1993) IP-10, a -C-X-C- chemokine, elicits a potent thymus dependent antitumor response in vivo. J Exp Med 178: 1057-1065.

Luster AD, Ravetch JV (1987) Genomic characterization of a gammainterferon inducible gene (IP-10) and identification of an interferoninducible hypersensitive site. Mol Cell Biol 7: 3723-3731.

Ma X, Norsworthy K, Kundu N, Rodgers WH, Gimotty PA, Goloubeva O, Lipsky M, Li Y, Holt D, Fulton A (2009) CXCR3 expression is associated with poor survival in breast cancer and promotes metastasis in a murine model. Mol Cancer Ther 8: 490-498.

Monteagudo C, Martin JM, Jorda E, Llombart-Bosch A (2007) CXCR3 chemokine receptor immunoreactivity in primary cutaneous malignant melanoma: correlation with clinicopathological prognostic factors. J Clin Pathol 60: 596-599.

Murakami T, Kawada K, Iwamoto M, Akagami M, Hida K, Nakanishi Y, Kanda K, Kawada M, Seno H, Taketo MM, Sakai Y (2013) The role of CXCR3 and CXCR4 in colorectal cancer metastasis. Int J Cancer 132: 276-287.

Qin S, Rottman JB, Myers P, Kassam N, Weinblatt M, Loetscher M, Koch AE, Moser B, Mackay CR (1998) The chemokine receptors CXCR3 and CCR5 mark subsets of $\mathrm{T}$ cells associated with certain inflammatory reactions J Clin Invest 101: 746-754.

Schultz J, Koczan D, Schmitz U, Ibrahim SM, Pilch D, Landsberg J, Kunz M (2010) Tumor-promoting role of signal transducer and activator of transcription (Stat)1 in late-stage melanoma growth. Clin Exp Metastasis 27: $133-140$.

Sistigu A, Yamazaki T, Vacchelli E, Chaba K, Enot DP, Adam J, Vitale I, Goubar A, Baracco EE, Remédios C, Fend L, Hannani D, Aymeric L, Ma Y, Niso-Santano M, Kepp O, Schultze JL, Tüting T, Belardelli F, Bracci L, La Sorsa V, Ziccheddu G, Sestili P, Urbani F, Delorenzi M, Lacroix-Triki M, Quidville V, Conforti R, Spano JP, Pusztai L, Poirier-Colame V, Delaloge S, Penault-Llorca F, Ladoire S, Arnould L, Cyrta J, Dessoliers MC, Eggermont A, Bianchi ME, Pittet M, Engblom C, Pfirschke C, Préville X, Uzè G, Schreiber RD, Chow MT, Smyth MJ, Proietti E, André F, Kroemer G, Zitvogel L (2014) Cancer cell-autonomous contribution of type I interferon signaling to the efficacy of chemotherapy. Nat Med 20: 1301-1309.

Smyth GK (2005) Limma: linear models for microarray data. In Bioinformatics and Computational Biology Solutions Using $R$ and Bioconductor, Gentleman R, Carey V, Dudoit S, Irizarry R, Huber W (eds), pp 397-420. Springer-Verlag: New York. 
Uppal A, Wightman SC, Mallon S, Oshima G, Pitroda SP, Zhang Q, Huang X, Darga TE, Huang L, Andrade J, Liu H, Ferguson MK, Greene GL, Posner MC, Hellman S, Khodarev NN, Weichselbaum RR (2015) 14q32-encoded microRNAs mediate an oligometastatic phenotype. Oncotarget 6: 3540-3552.

Utsumi T, Suyama T, Imamura Y, Fuse M, Sakamoto S, Nihei N, Ueda T, Suzuki H, Seki N, Ichikawa T (2014) The association of CXCR3 and renal cell carcinoma metastasis. J Urol 192: 567-574.

Walser TC, Rifat S, Ma X, Kundu N, Ward C, Goloubeva O, Johnson MG, Medina JC, Collins TL, Fulton AM (2006) Antagonism of CXCR3 inhibits lung metastasis in a murine model of metastatic breast cancer. Cancer Res 66: 7701-7707.

Weichselbaum RR, Ishwaran H, Yoon T, Nuyten DS, Baker SW, Khodarev N, Su AW, Shaikh AY, Roach P, Kreike B, Roizman B, Bergh J, Pawitan Y, van de Vijver MJ, Minn AJ (2008) An interferon-related gene signature for DNA damage resistance is a predictive marker for chemotherapy and radiation for breast cancer. Proc Natl Acad Sci USA 105: 18490-18495.

Wennerberg E, Kremer V, Childs R, Lundqvist A (2014) CXCL10-induced migration of adoptively transferred human natural killer cells toward solid tumors causes regression of tumor growth in vivo. Cancer Immunol Immunother 64(2): 225-235.

Wenzel J, Bekisch B, Uerlich M, Haller O, Bieber T, Tüting T (2005) Type I interferon-associated recruitment of cytotoxic lymphocytes: a common mechanism in regressive melanocytic lesions. Am J Clin Pathol 124: 37-48.

Wu Q, Dhir R, Wells A (2012) Altered CXCR3 isoform expression regulates prostate cancer cell migration and invasion. Mol Cancer 11: 3 .

Zipin-Roitman A, Meshel T, Sagi-Assif O, Shalmon B, Avivi C, Pfeffer RM, Witz IP, Ben Baruch A (2007) CXCL10 promotes invasion-related properties in human colorectal carcinoma cells. Cancer Res 67: 3396-3405.

Zlotnik A, Yoshie O (2012) The chemokine superfamily revisited. Immunity 36: 705-716.

This work is published under the standard license to publish agreement. After 12 months the work will become freely available and the license terms will switch to a Creative Commons AttributionNonCommercial-Share Alike 4.0 Unported License

Supplementary Information accompanies this paper on British Journal of Cancer website (http://www.nature.com/bjc) 